\title{
Beginning Oracle \\ Application Express 5
}

Doug Gault 


\title{
Beginning Oracle Application Express 5
}

\author{
Copyright $\odot 2015$ by Doug Gault
}

This work is subject to copyright. All rights are reserved by the Publisher, whether the whole or part of the material is concerned, specifically the rights of translation, reprinting, reuse of illustrations, recitation, broadcasting, reproduction on microfilms or in any other physical way, and transmission or information storage and retrieval, electronic adaptation, computer software, or by similar or dissimilar methodology now known or hereafter developed. Exempted from this legal reservation are brief excerpts in connection with reviews or scholarly analysis or material supplied specifically for the purpose of being entered and executed on a computer system, for exclusive use by the purchaser of the work. Duplication of this publication or parts thereof is permitted only under the provisions of the Copyright Law of the Publisher's location, in its current version, and permission for use must always be obtained from Springer. Permissions for use may be obtained through RightsLink at the Copyright Clearance Center. Violations are liable to prosecution under the respective Copyright Law.

ISBN-13 (pbk): 978-1-4842-0467-2

ISBN-13 (electronic): 978-1-4842-0466-5

Trademarked names, logos, and images may appear in this book. Rather than use a trademark symbol with every occurrence of a trademarked name, logo, or image we use the names, logos, and images only in an editorial fashion and to the benefit of the trademark owner, with no intention of infringement of the trademark.

The use in this publication of trade names, trademarks, service marks, and similar terms, even if they are not identified as such, is not to be taken as an expression of opinion as to whether or not they are subject to proprietary rights.

While the advice and information in this book are believed to be true and accurate at the date of publication, neither the authors nor the editors nor the publisher can accept any legal responsibility for any errors or omissions that may be made. The publisher makes no warranty, express or implied, with respect to the material contained herein.

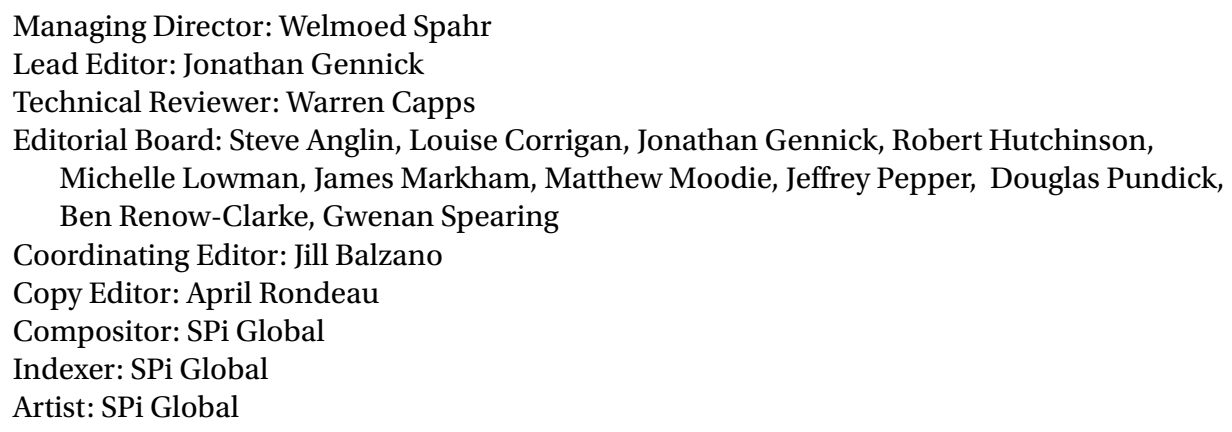

Distributed to the book trade worldwide by Springer Science+Business Media New York, 233 Spring Street, 6th Floor, New York, NY 10013. Phone 1-800-SPRINGER, fax (201) 348-4505, email orders-ny@springer-sbm.com, or visit www.springeronline.com. Apress Media, LLC is a California LLC and the sole member (owner) is Springer Science + Business Media Finance Inc (SSBM Finance Inc). SSBM Finance Inc is a Delaware corporation.

For information on translations, please e-mail rights@apress.com, or visit www. apress .com.

Apress and friends of ED books may be purchased in bulk for academic, corporate, or promotional use. eBook versions and licenses are also available for most titles. For more information, reference our Special Bulk Sales-eBook Licensing web page at www. apress. com/bulk-sales.

Any source code or other supplementary material referenced by the author in this text is available to readers at www. apress.com/. For detailed information about how to locate your book's source code, go to www. apress.com/source-code/. 
To those in search of knowledge and better understanding,

I dedicate this effort. Hopefully, as your skills grow,

you too will continue to share the wealth.

-Doug Gault 



\section{Contents at a Glance}

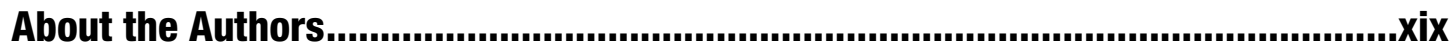

About the Technical Reviewer .........................................................................xxi

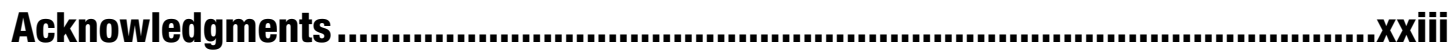

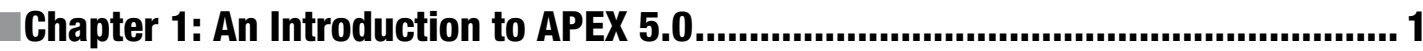

Chapter 2: A Developer's Overview ................................................................ 7

Chapter 3: Identifying the Problem and Designing the Solution......................... 37

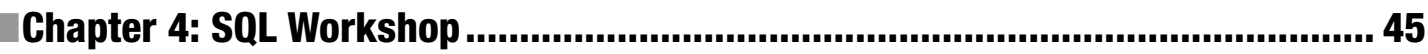

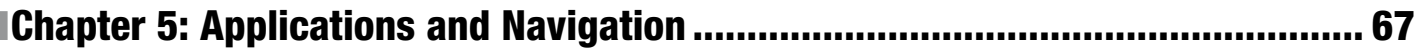

Chapter 6: Forms and Reports: The Basics ........................................................ 107

Chapter 7: Forms and Reports: Advanced ..................................................... 167

Chapter 8: Programmatic Elements ............................................................................ 231

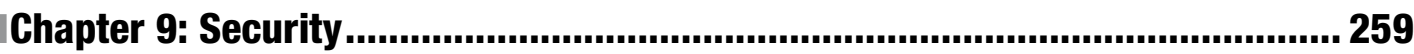

Chapter 10: Application Bundling and Deployment....................................... 287

Chapter 11: Understanding Websheets .................................................... 309

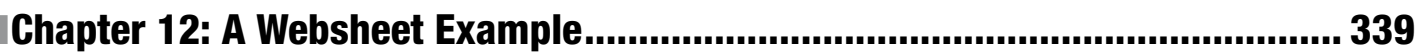

Chapter 13: Extended Developer Tools ...................................................... 359

Chapter 14: Managing Workspaces............................................................... 383 
CONTENTS AT A GLANCE

Chapter 15: Team Development................................................................... 401

Chapter 16: Dynamic Actions .......................................................................... 431

Appendix A: Page Designer Walkthrough and Reference ................................... 449

Index 


\section{Contents}

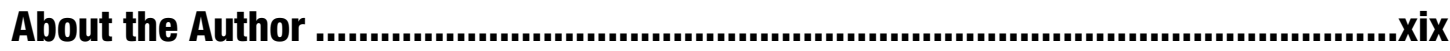

About the Technical Reviewer ...............................................................................

Acknowledgments ....................................................................................xiii

Chapter 1: An Introduction to APEX 5.0......................................................... 1

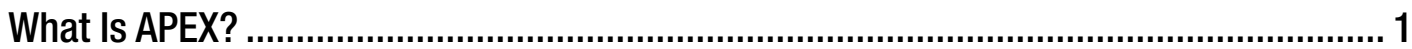

A Brief History of APEX ................................................................................... 2

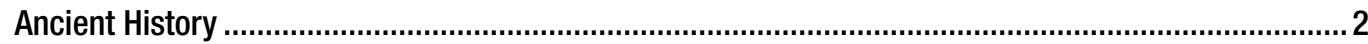

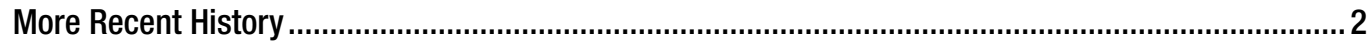

APEX 5.0 and the Future

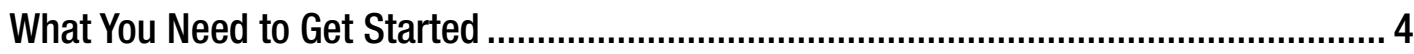

Access to an APEX Instance .............................................................................................

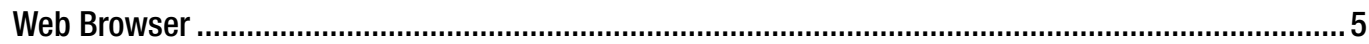

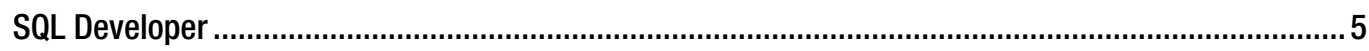

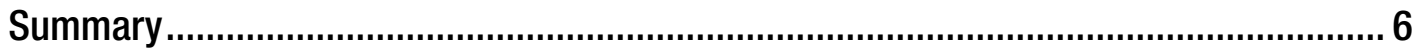

Chapter 2: A Developer's Overview ........................................................................ 7

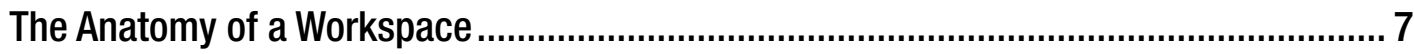

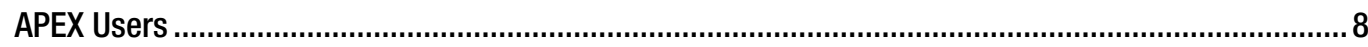

Applications, Pages, Regions, and Items ............................................................................. 9

Workspaces, Applications, and Schemas ....................................................................................10

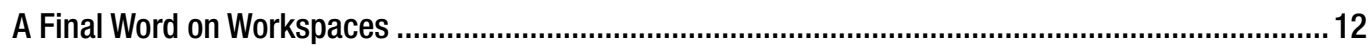

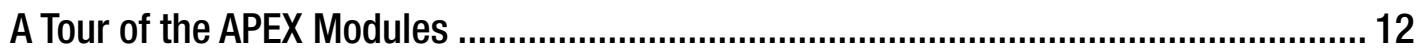

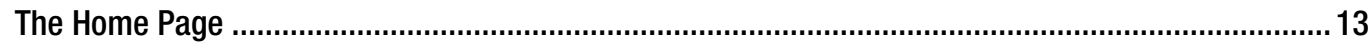

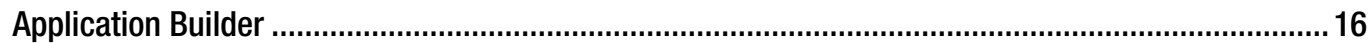




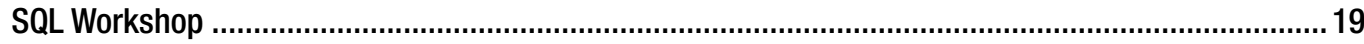

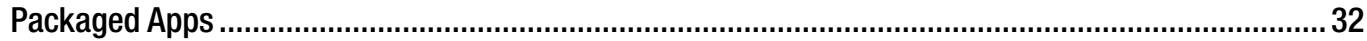

Administration and Team Development..................................................................................... 35

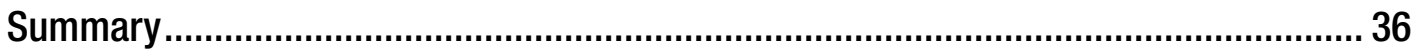

Chapter 3: Identifying the Problem and Designing the Solution........................ 37

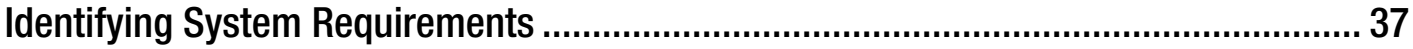

Never a Clean Slate

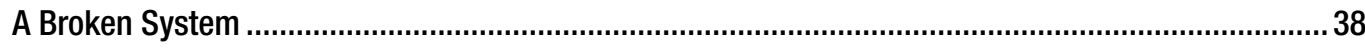

How Do You Fix Things? ......................................................................................................... 38

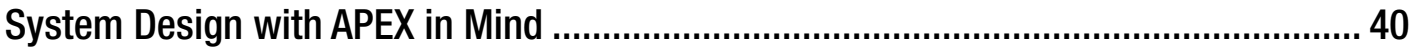

Table Definition and User-Interface Defaults................................................................................ 40

APEX and Primary Keys ................................................................................................... 41

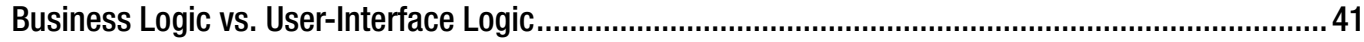

Placement of Database Objects.............................................................................................. 42

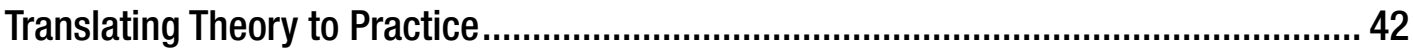

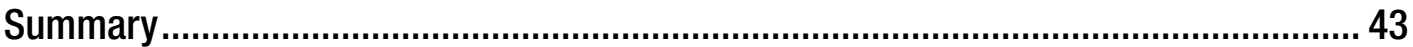

Chapter 4: SQL Workshop.............................................................................. 45

Creating Objects with the Object Browser .......................................................... 45

Loading Data with the Data Workshop Utility ......................................................... 52

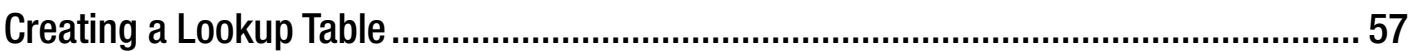

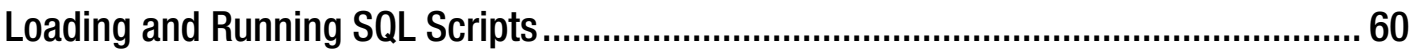

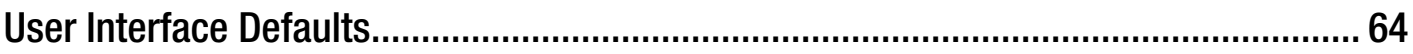

Understanding User Interface Defaults.......................................................................................... 64

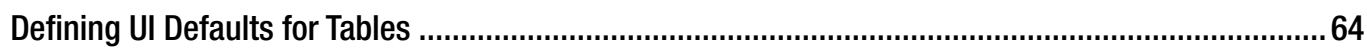

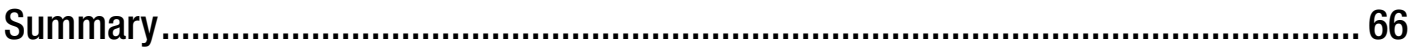

Chapter 5: Applications and Navigation .................................................. 67

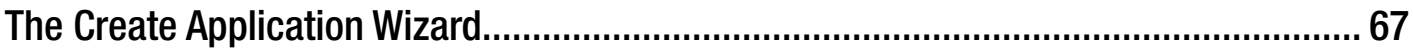

Sample and Packaged Applications ...................................................................................... 68

Websheet Applications ........................................................................................................72 


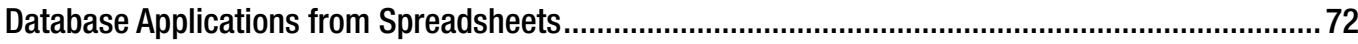

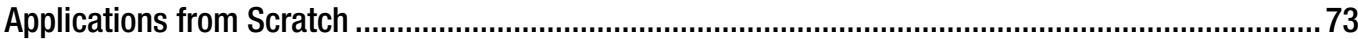

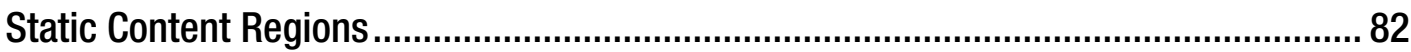

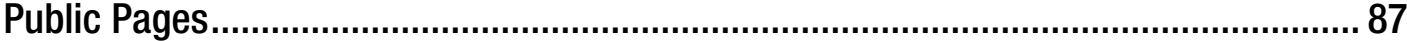

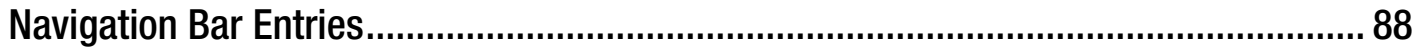

Global Pages............................................................................................................... 91

Breadcrumb Regions.......................................................................................... 93

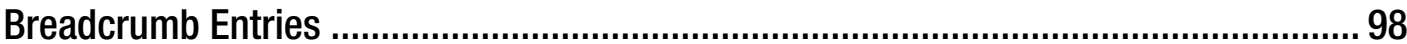

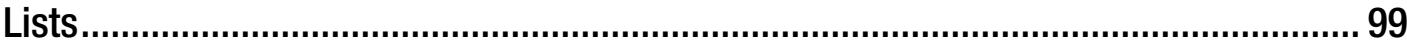

Lists of Values

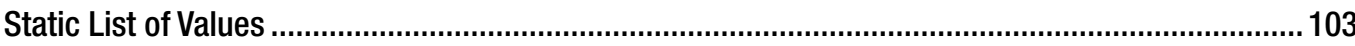

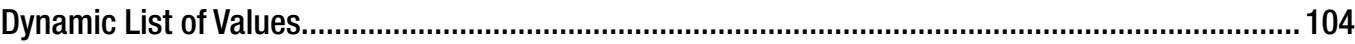

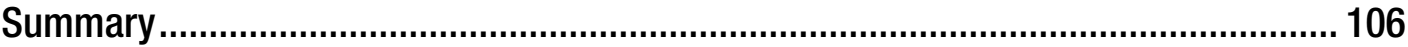

Chapter 6: Forms and Reports: The Basics .................................................. 107

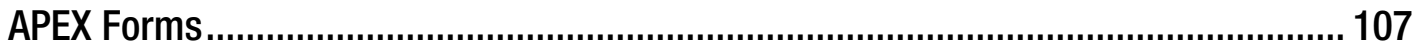

Form on a Table.................................................................................................... 109

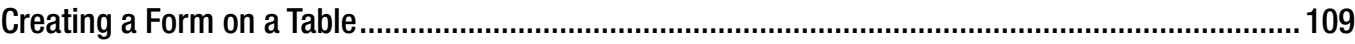

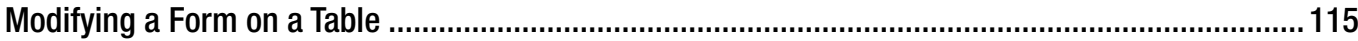

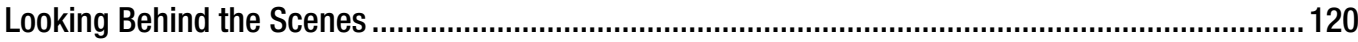

Form on a Procedure......................................................................................... 122

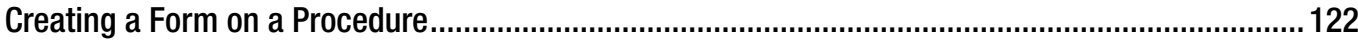

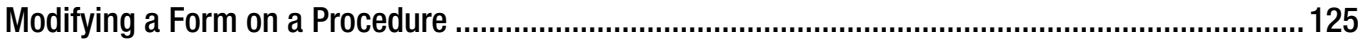

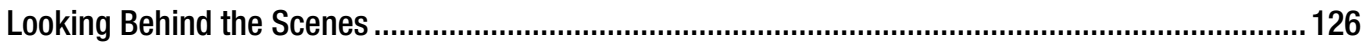

Master-Detail Report and Form ...................................................................... 127

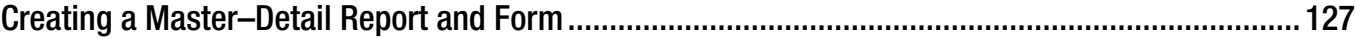

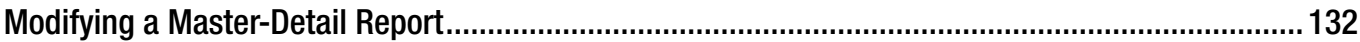

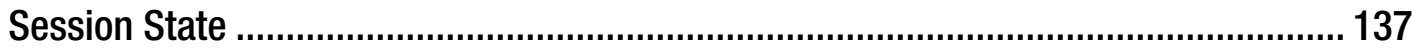

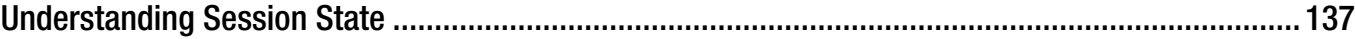

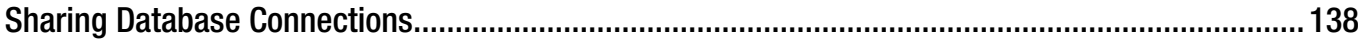




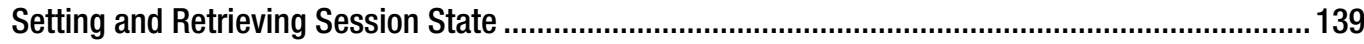

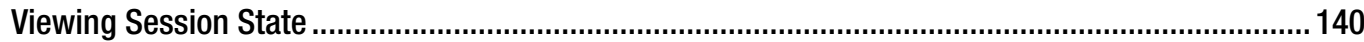

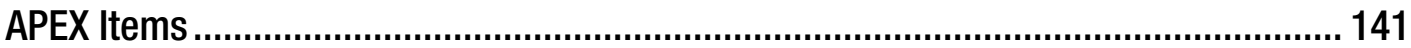

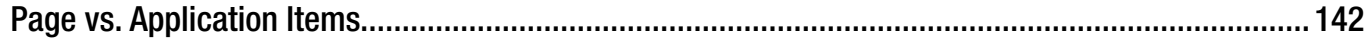

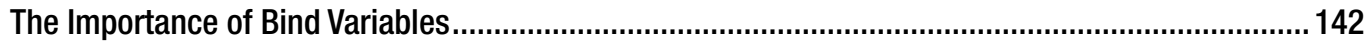

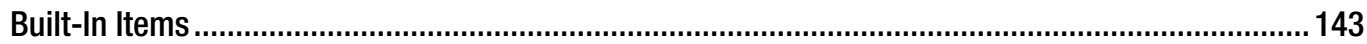

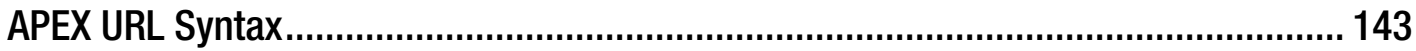

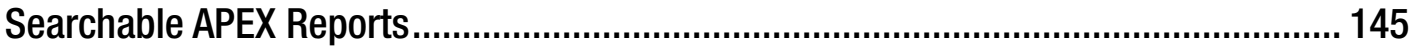

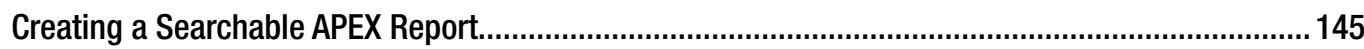

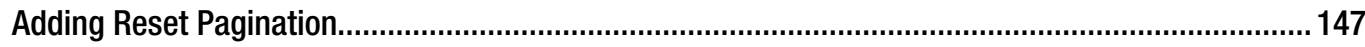

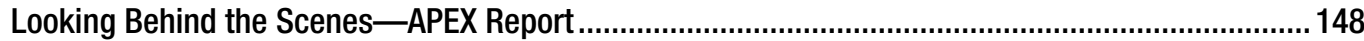

Looking Behind the Scenes_APEX Master-Detail Forms ........................................................... 150

More on APEX Forms ........................................................................................... 152

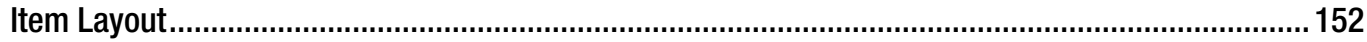

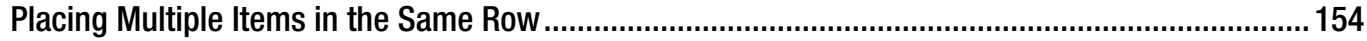

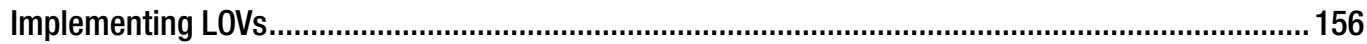

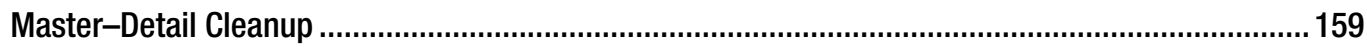

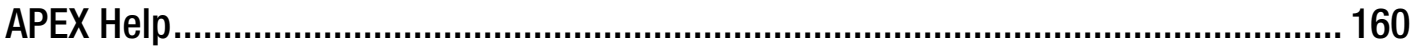

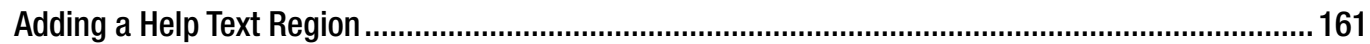

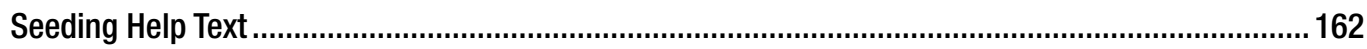

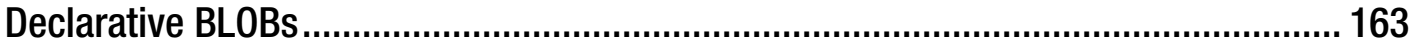

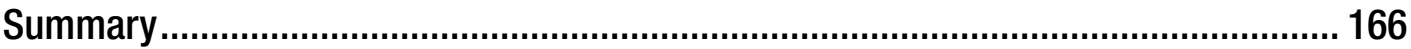

Chapter 7: Forms and Reports: Advanced ................................................ 167

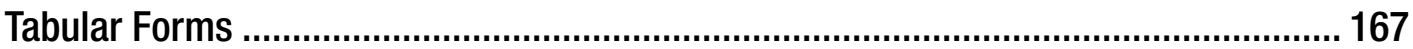

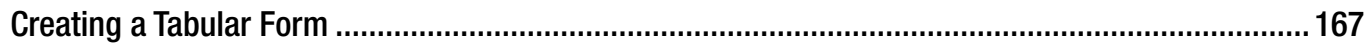

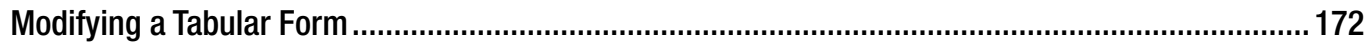

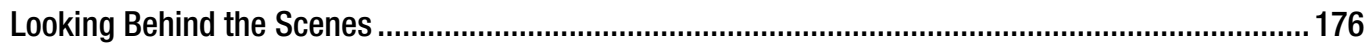

Interactive Reports ................................................................................... 177

Creating an Interactive Report................................................................................................. 177

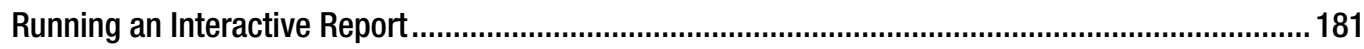




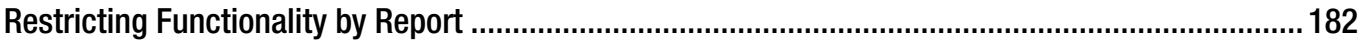

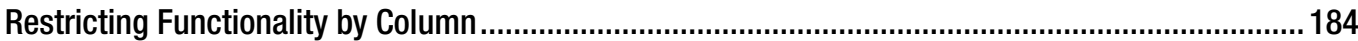

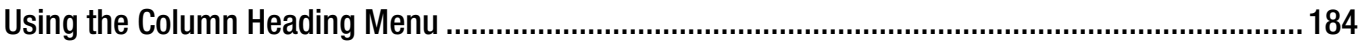

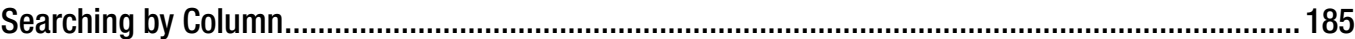

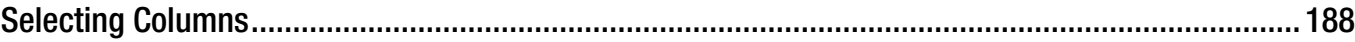

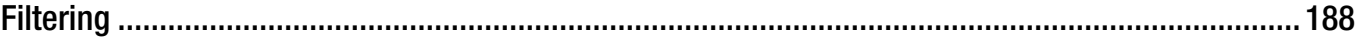

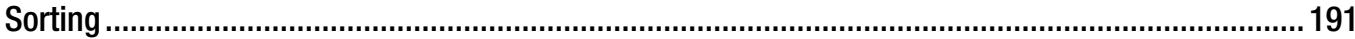

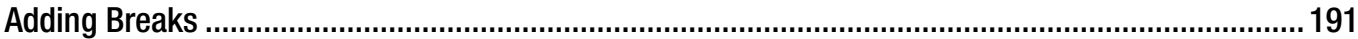

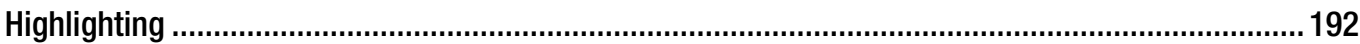

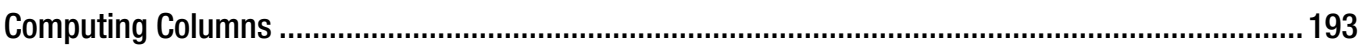

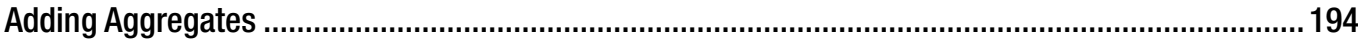

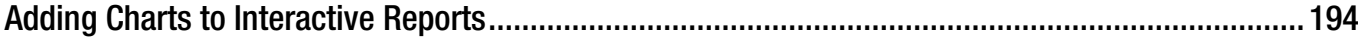

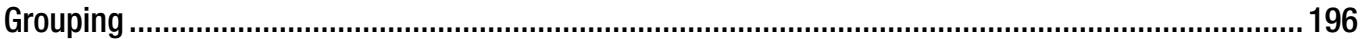

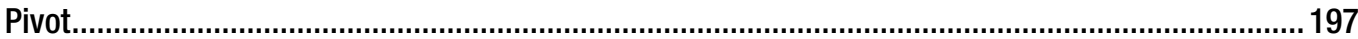

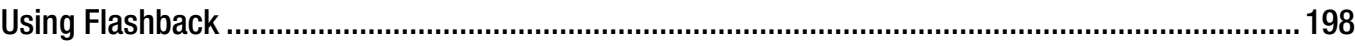

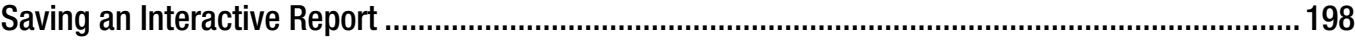

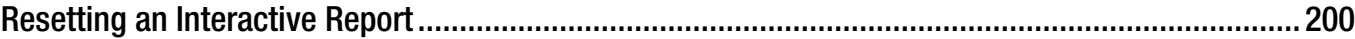

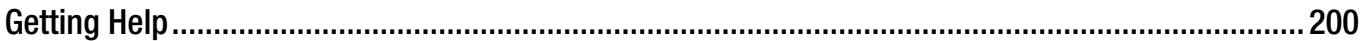

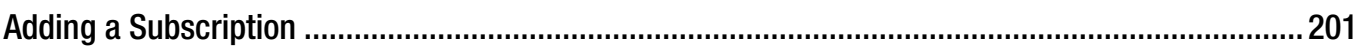

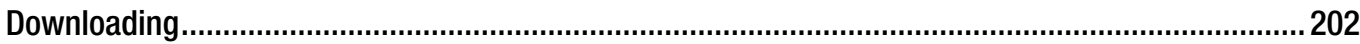

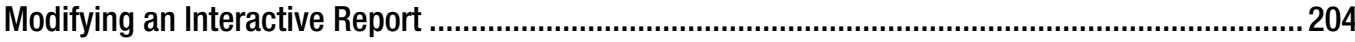

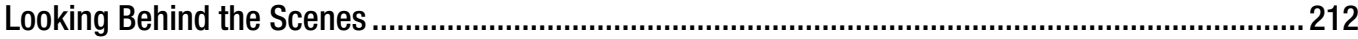

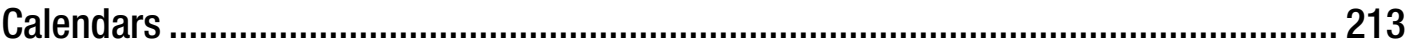

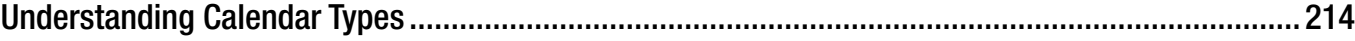

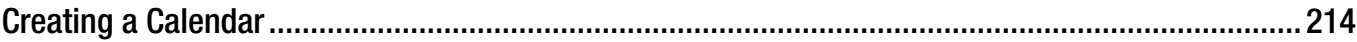

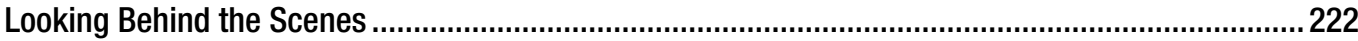

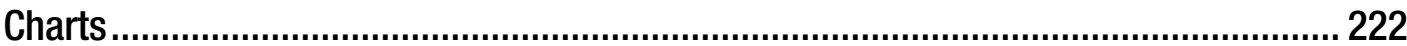

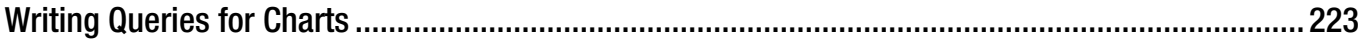

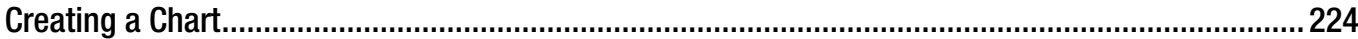




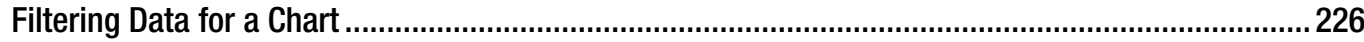

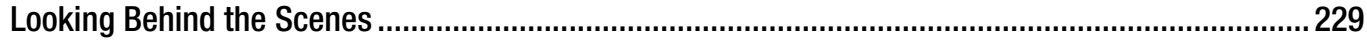

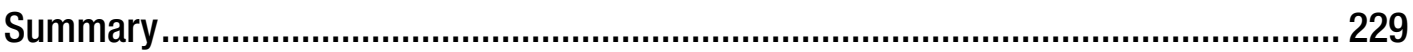

Chapter 8: Programmatic Elements .................................................... 231

Conditions ........................................................................................................ 231

Required Values.................................................................................................. 231

Validations................................................................................................................ 234

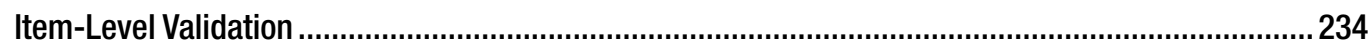

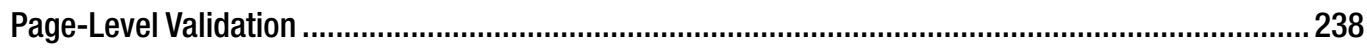

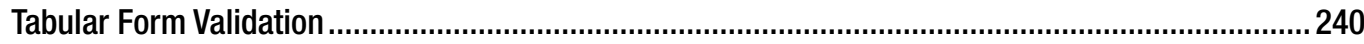

Computations .................................................................................................. 242

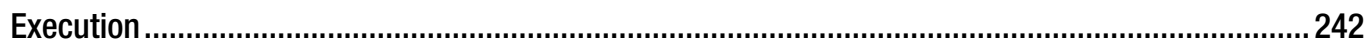

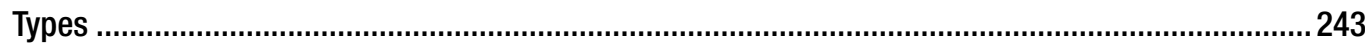

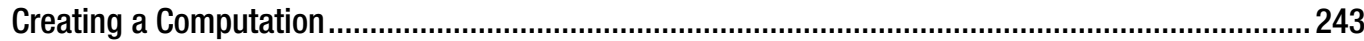

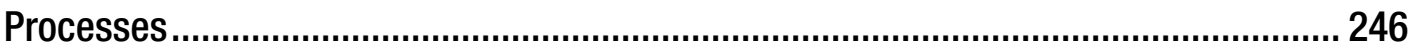

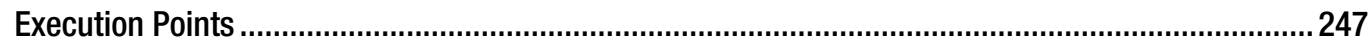

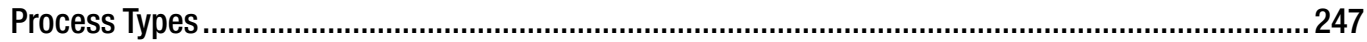

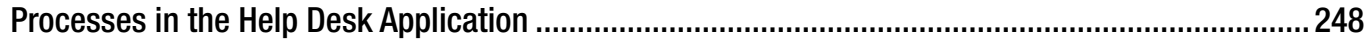

PL/SQL Regions .................................................................................. 251

Dynamic SQL .................................................................................................... 253

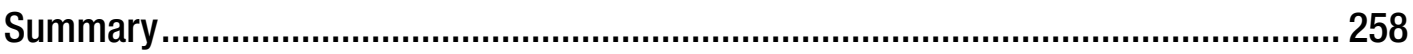

Chapter 9: Security........................................................................... 259

User-Maintenance Navigation ............................................................................. 259

User-Maintenance Data Entry ................................................................................ 263

Authentication .......................................................................................................... 269

Custom Authentication Schemes ....................................................................... 270

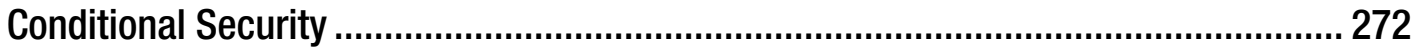

Access Control .......................................................................................... 273

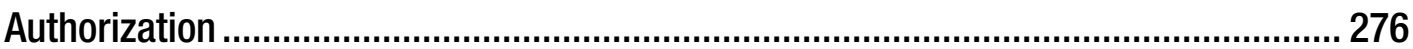




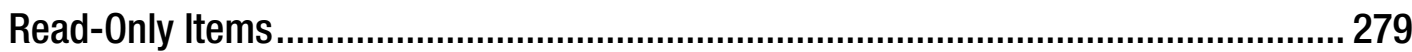

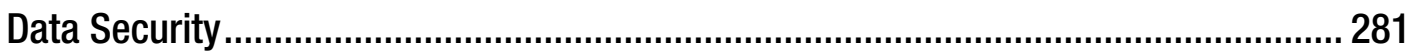

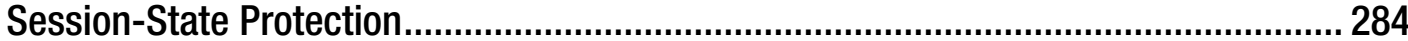

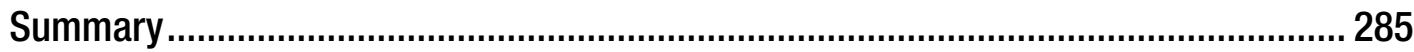

Chapter 10: Application Bundling and Deployment........................................ 287

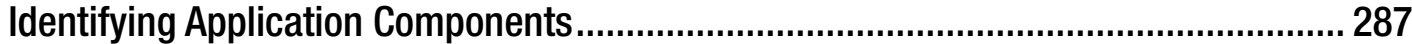

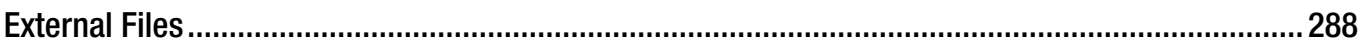

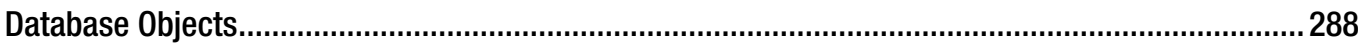

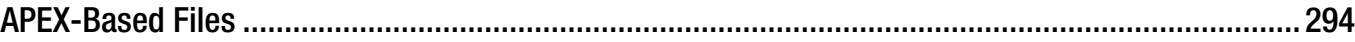

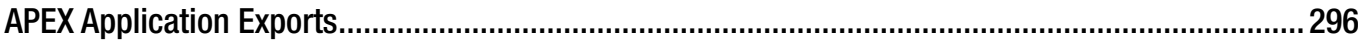

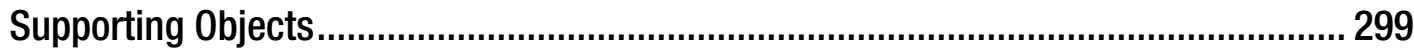

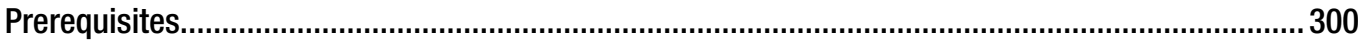

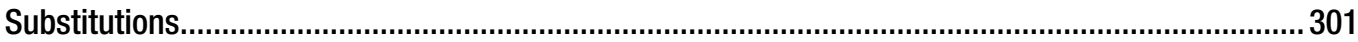

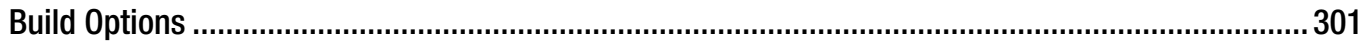

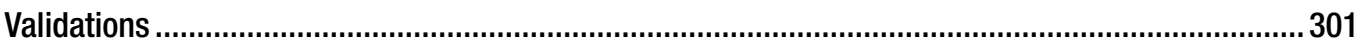

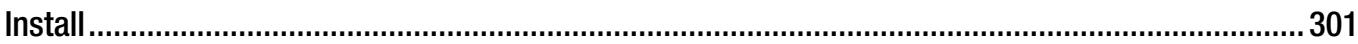

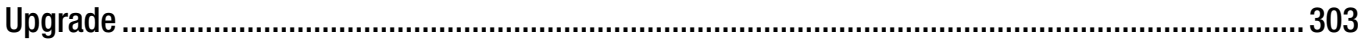

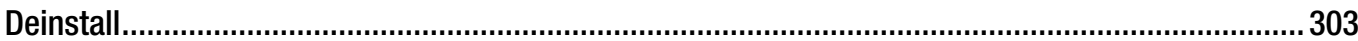

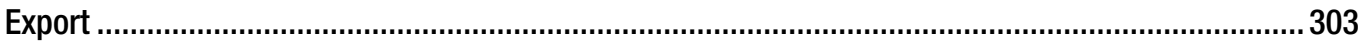

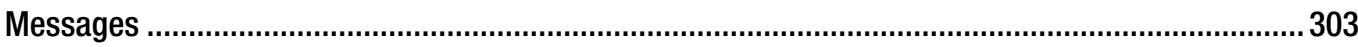

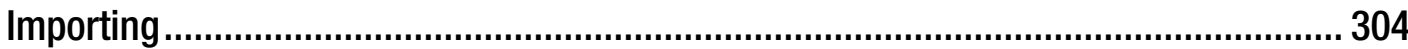

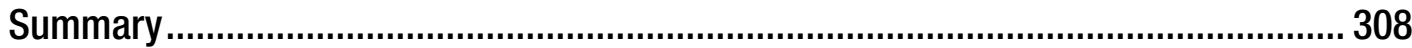

Chapter 11: Understanding Websheets ........................................................ 309

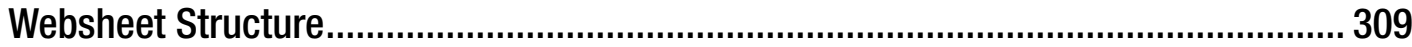

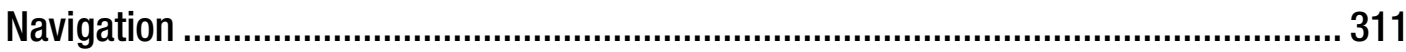

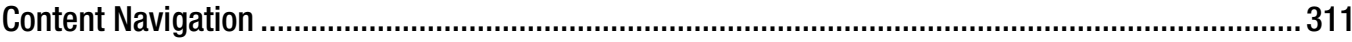

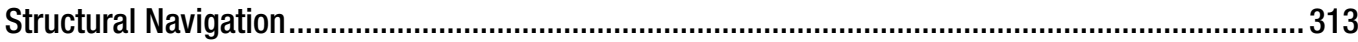

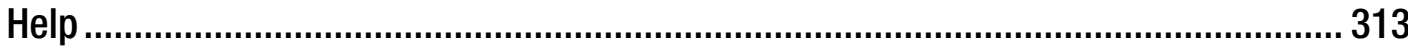

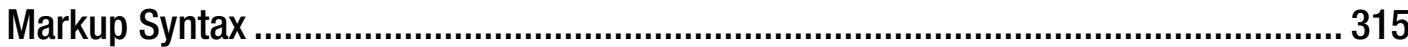




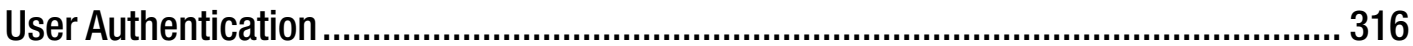

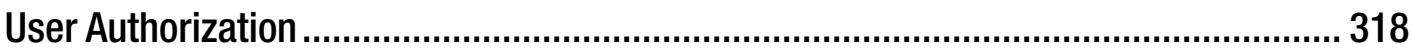

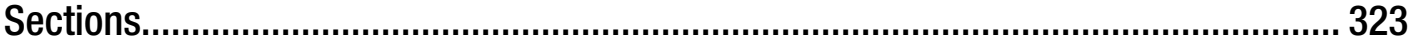

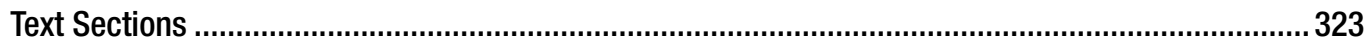

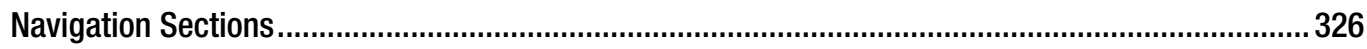

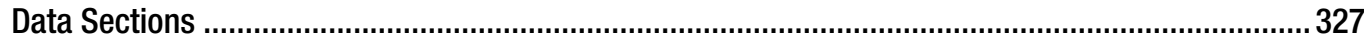

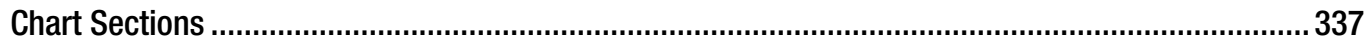

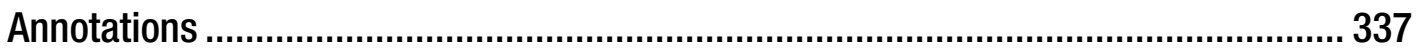

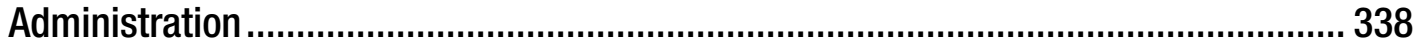

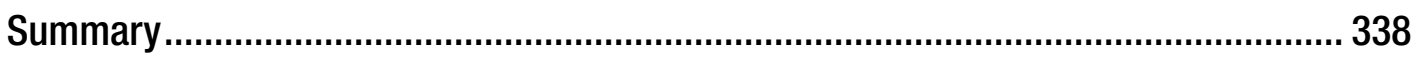

Chapter 12: A Websheet Example................................................................... 339

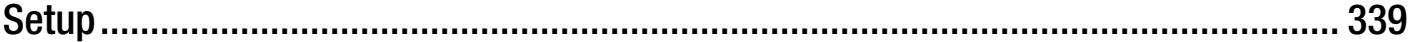

Creating and Configuring a Websheet Application .................................................... 340

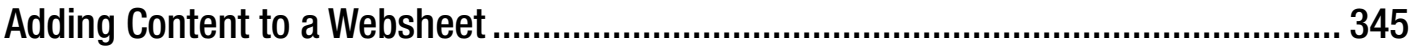

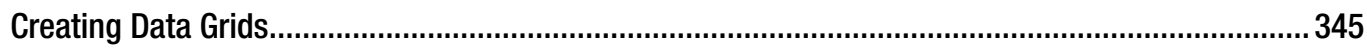

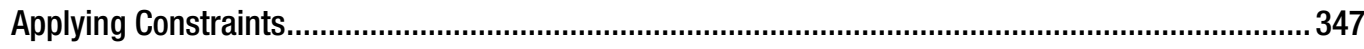

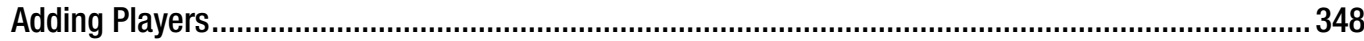

Creating Alternate Default Reports ....................................................................................... 349

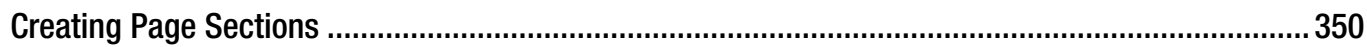

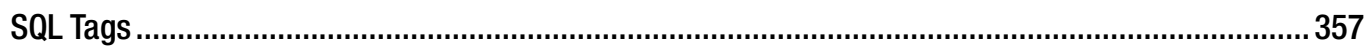

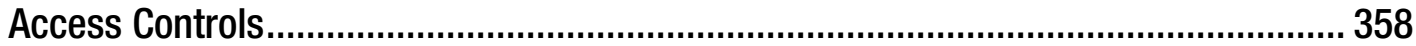

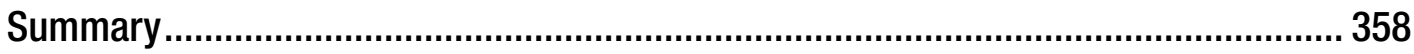

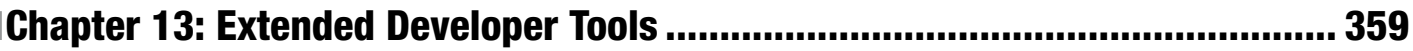

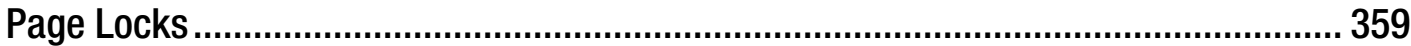

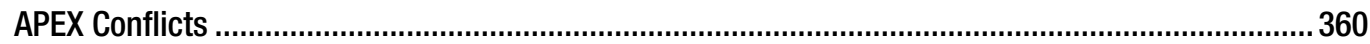

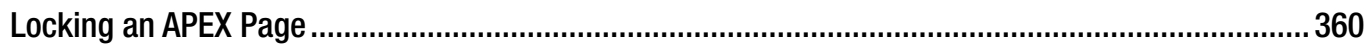

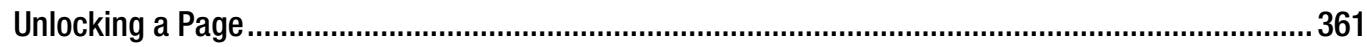

Administering Page Locks ................................................................................................. 361 


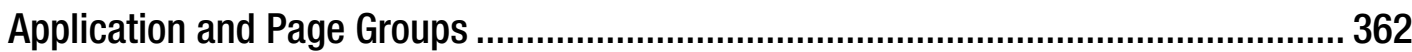

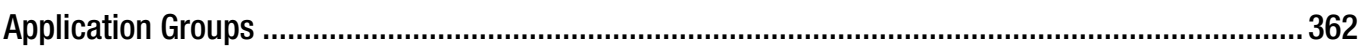

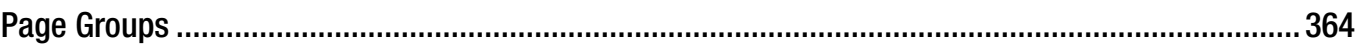

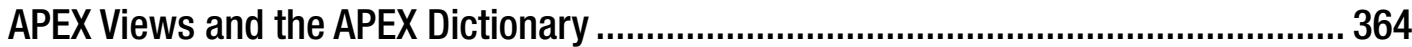

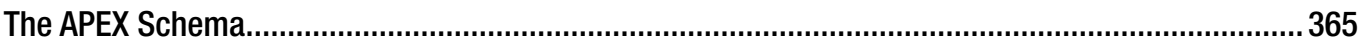

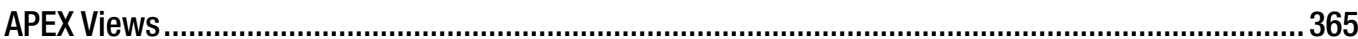

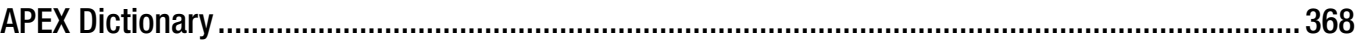

Searching in APEX

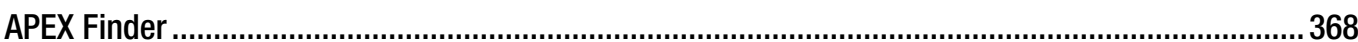

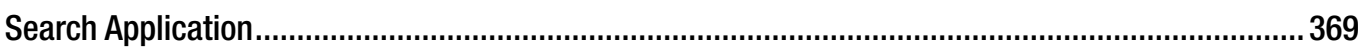

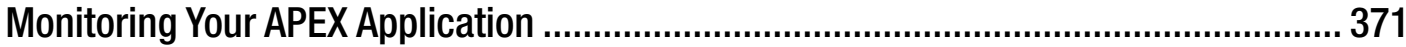

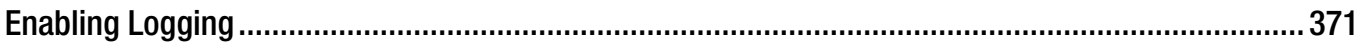

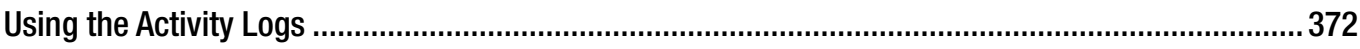

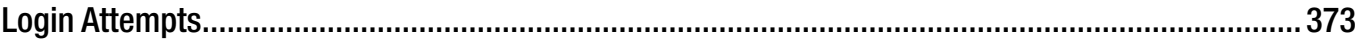

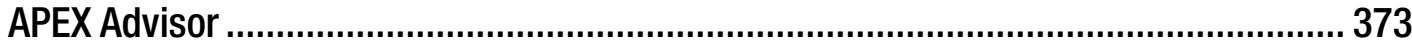

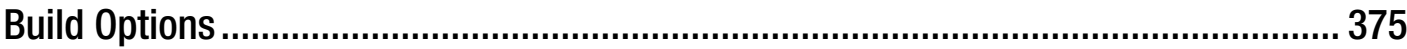

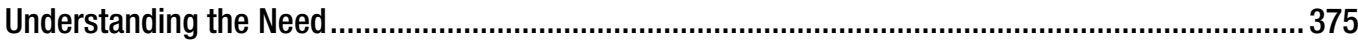

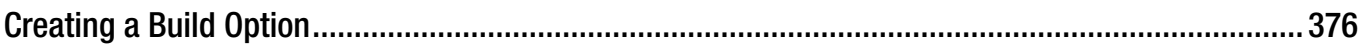

Configuring Build Options .................................................................................................. 377

Prompting for Build Option Status ......................................................................................... 377

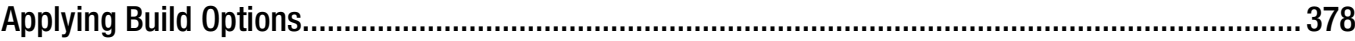

Reporting on Build Option Utilization...................................................................................... 379

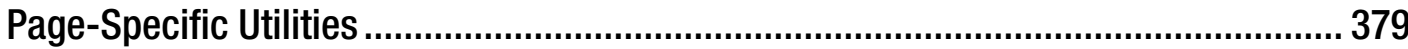

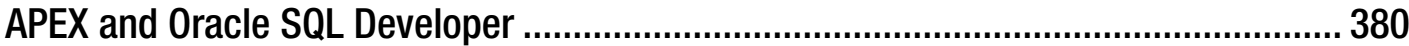

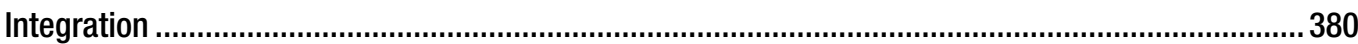

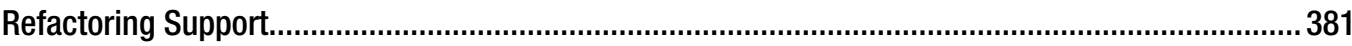

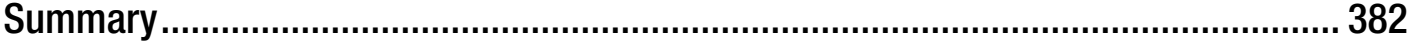


Chapter 14: Managing Workspaces................................................................. 383

Learning About Your Environment .......................................................................... 383

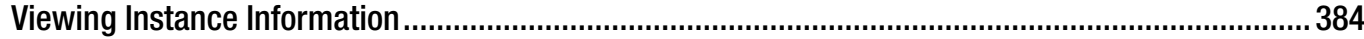

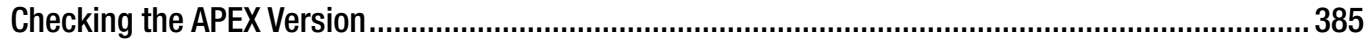

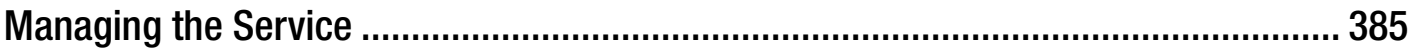

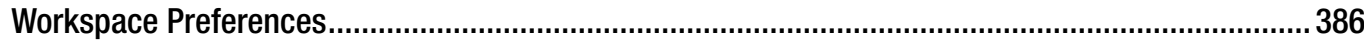

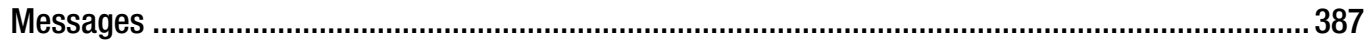

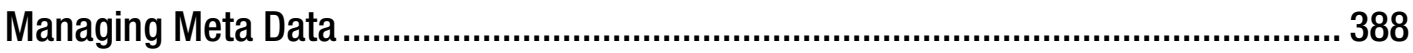

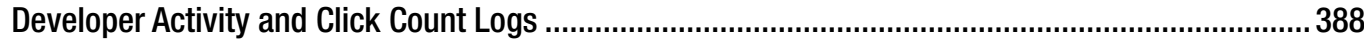

Session State

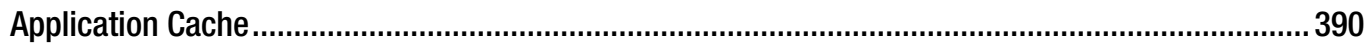

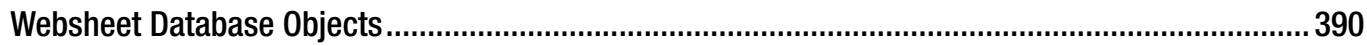

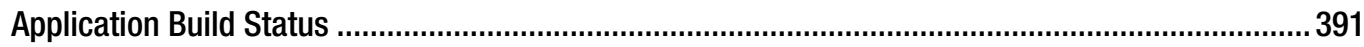

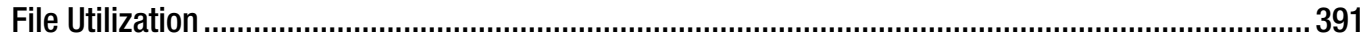

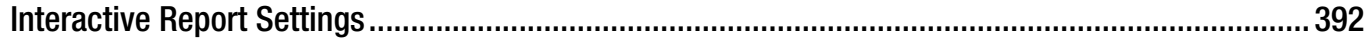

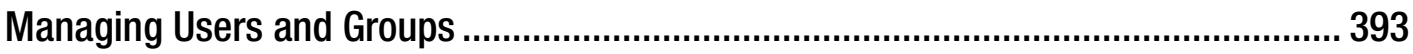

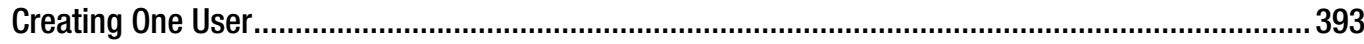

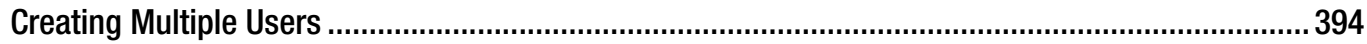

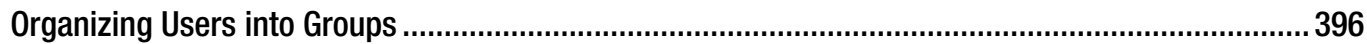

Viewing Usage Reports and Dashboards ............................................................. 399

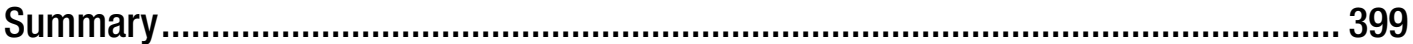

Chapter 15: Team Development......................................................................... 401

Team Development Overview............................................................................ 401

Team Development Interface ............................................................................. 403

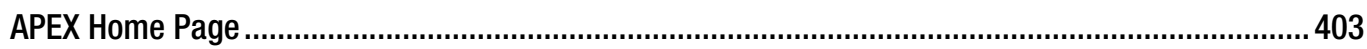

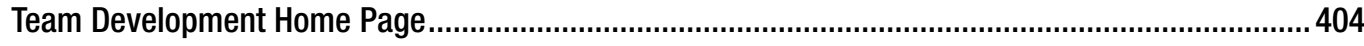

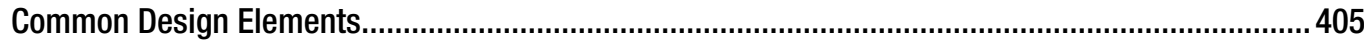

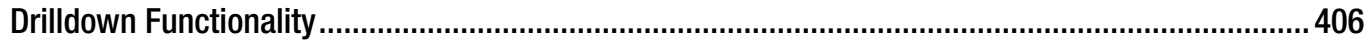

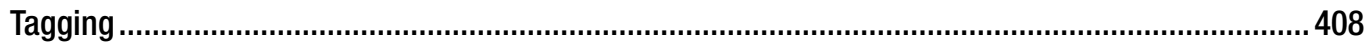




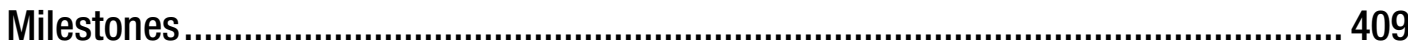

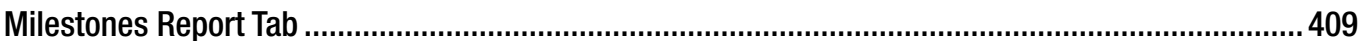

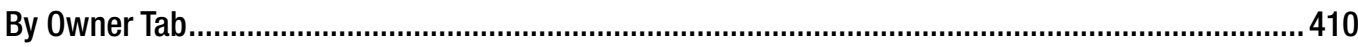

Features by Milestone Tab ................................................................................................... 410

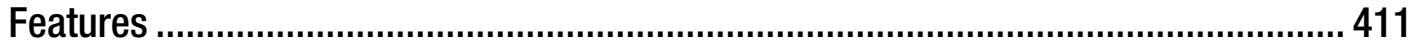

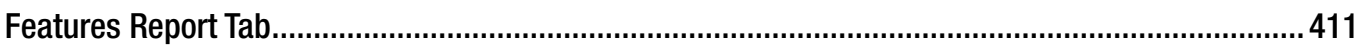

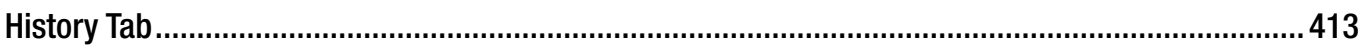

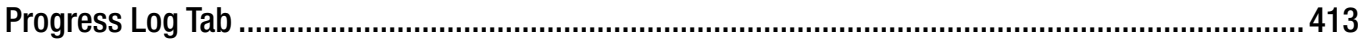

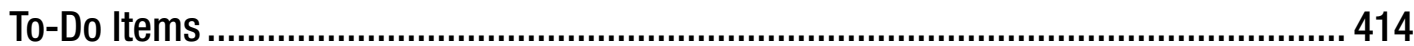

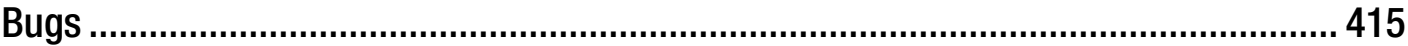

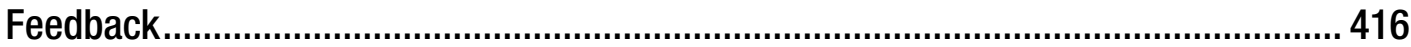

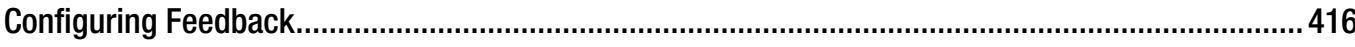

Polishing the Feedback Page .............................................................................................. 419

Viewing Feedback ............................................................................................................ 423

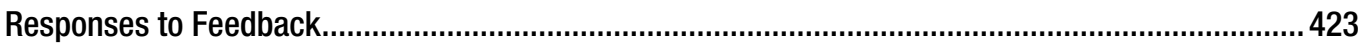

Communication Between Workspaces ...................................................................................... 423

Team Development Utilities.................................................................................... 424

Team Development Settings............................................................................................... 424

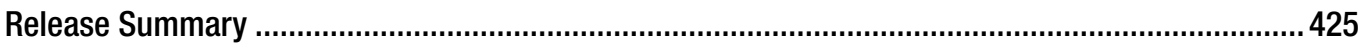

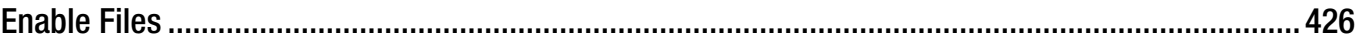

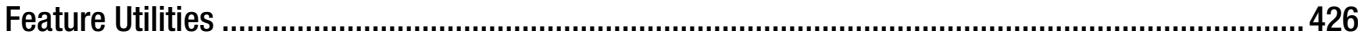

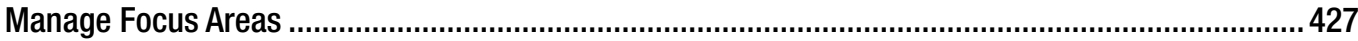

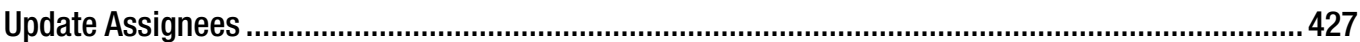

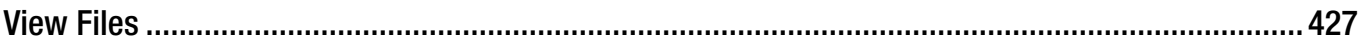

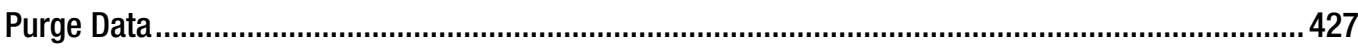

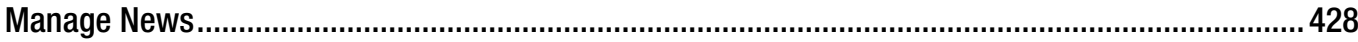

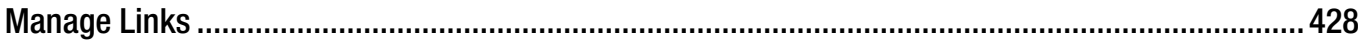

User Roles for Team Development ..................................................................... 429

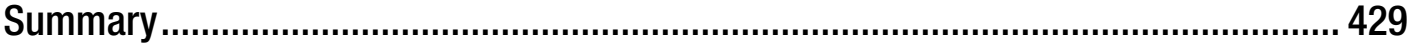


Chapter 16: Dynamic Actions ..................................................................... 431

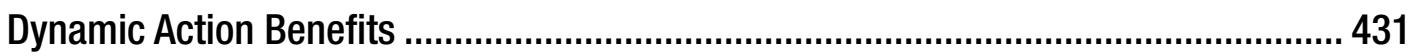

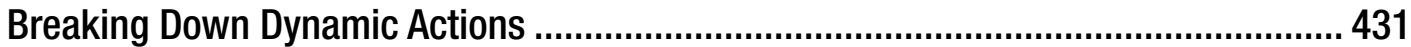

Dynamic Actions in the Help Desk Application ........................................................ 432

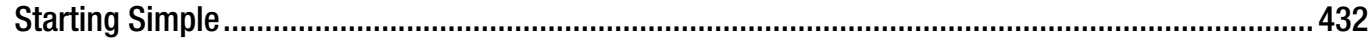

Using Page-Level Events ...................................................................................................... 439

Dynamic Actions with Multiple Triggering Elements ................................................................ 441

Dynamic Actions Using PL/SQL.......................................................................................... 443

Dynamic Actions Using JavaScript ......................................................................................... 445

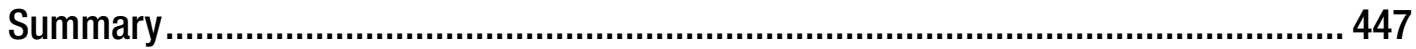

Appendix A: Page Designer Walkthrough and Reference ................................ 449

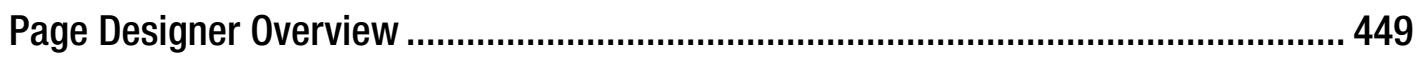

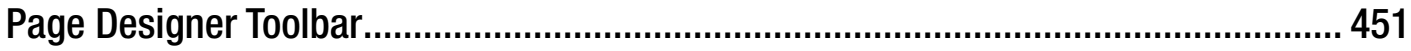

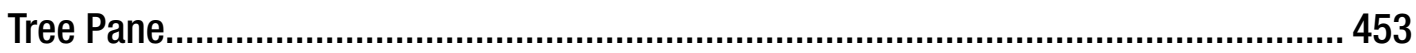

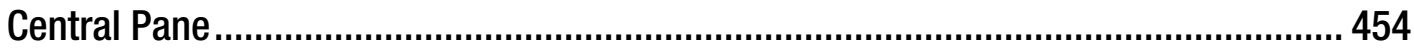

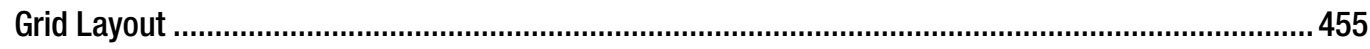

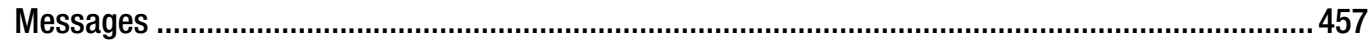

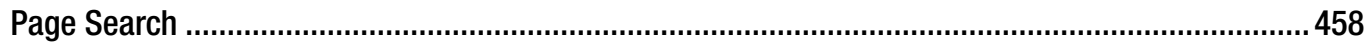

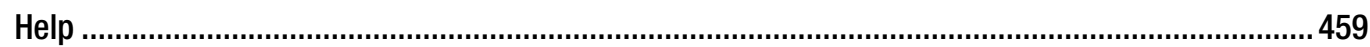

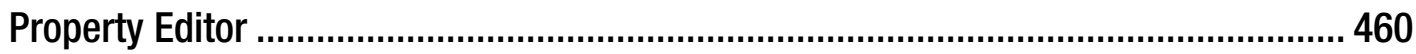

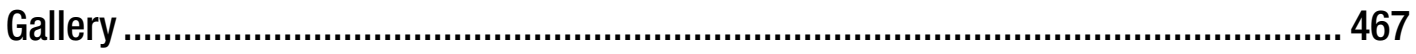

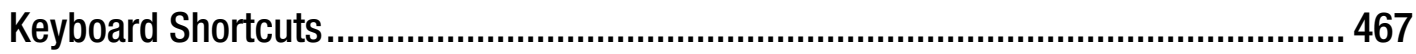

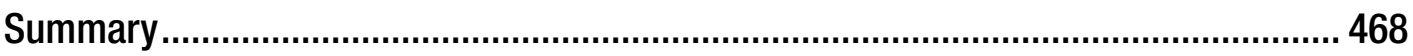

Index 


\section{About the Author}

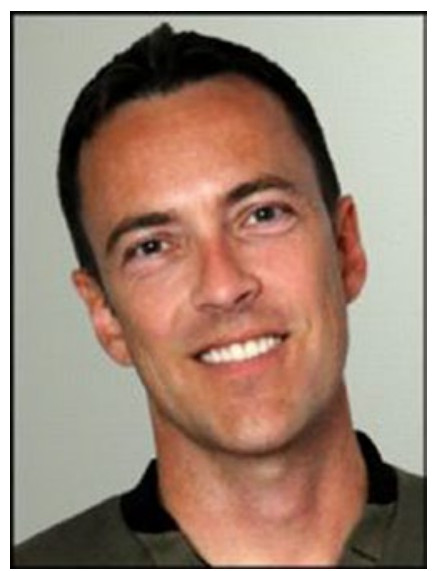

Doug Gault is a Consulting Member of Technical Staff at Oracle Corporation and has been working with Oracle since 1988, starting with version 5.1B, SQL*Forms 2.0, and RPT/RPF. He has focused his career on Oracle's development technologies, spending the majority of that time dedicated to web-based technologies, including the OWA Web Toolkit, PL/SQL Server Pages, WebDB, Oracle Portal, and more recently HTML-DB and APEX.

His many years of Oracle experience have taken him all over the world to participate in some truly groundbreaking projects. Doug has presented and participated in roundtable discussions at a number of conferences, including Oracle OpenWorld, UKOUG, and ODTUG's APEXposed \& Kaleidoscope conferences. He holds an Associate's Degree in Computer Science and an honorary Master's Degree from The School of Hard Knocks, believing there is no replacement for hard-earned experience.

Doug can be found on Twitter as @dgault_apex and on his blog at douggault.blogspot.com. 



\section{About the Technical Reviewer}

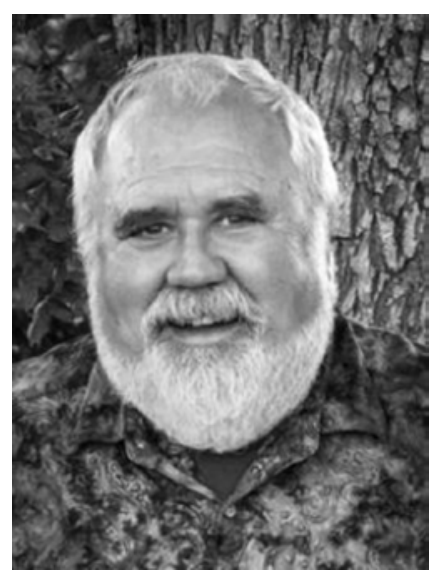

Warren Capps, president of Illuminations Inc, has worked with Oracle since 1987 when he worked on version 5.1a. Since 1991, his principal efforts have been spent in training clients in the use of Oracle products, concentrating on database server technologies. He is a well-known presenter at user-group conferences and has written numerous articles and book reviews for a variety of publications. He also ran an Oracle bookstore for ten years and is a retired Navy Commander.

When not teaching, Warren has myriad activities to keep him busy. He is an avid photographer and has run photography workshops in southern New Mexico. His photography has led him to visit over 25 countries. Additionally, he plays classical guitar, collects coins, and loves to travel the country with his wife and cat. He is currently a resident of Austin, Texas. 



\section{Acknowledgments}

First, my heart-felt thanks to all the co-authors of the original version of this book: Karen Cannell, Patrick Cimolini, Martin D'Souza, and Tim St. Hilaire. Warren Capps also needs to be thanked for his technical review efforts and his input on content and form. If not for these wonderful people, this book may never have come to be. The opportunity to work with such a talented and distinguished group of individuals has been a pleasure.

I'd also like to thank a few people who have been driving forces in my life: Kerry Osborne for providing me with an immense amount of mentorship and encouragement over the years, even after having left his employ; Cary Millsap for his friendship and helping to solidify in my mind how to think objectively about technology and to use proof to find the truth; and last but not least, Scott Spendolini for his all-around support before, during, and after the book. Without these people, I wouldn't be where I am today.

—Doug Gault 